\title{
Evaluating the yield of systematic screening for tuberculosis among three priority groups in Ho Chi Minh City, Viet Nam
}

Luan Nguyen Quang Vo ${ }^{1,2^{*}} \mathbb{D}$, Andrew James Codlin ${ }^{1}$, Rachel Jeanette Forse ${ }^{1}$, Nga Thuy Nguyen ${ }^{1}$, Thanh Nguyen Vu ${ }^{3}$, Giang Truong Le ${ }^{3}$, Vinh Van Truong ${ }^{4}$, Giang Chau Do ${ }^{4}, \mathrm{Ha} \mathrm{Minh} \mathrm{Dang}^{4}$, Lan Huu Nguyen ${ }^{4}$, Hoa Binh Nguyen ${ }^{5}$, Nhung Viet Nguyen ${ }^{5}$, Jens Levy ${ }^{6}$, Knut Lonnroth7, S. Bertel Squire ${ }^{8}$ and Maxine Caws ${ }^{8,9}$

\begin{abstract}
Background: In order to end tuberculosis (TB), it is necessary to expand coverage of TB care services, including systematic screening initiatives. However, more evidence is needed for groups among whom systematic screening is only conditionally recommended by the World Health Organization. This study evaluated concurrent screening in multiple target groups using community health workers (CHW).

Methods: In our two-year intervention study lasting from October 2017 to September 2019, CHWs in six districts of Ho Chi Minh City, Viet Nam verbally screened three urban priority groups: (1) household TB contacts; (2) close TB contacts; and (3) residents of urban priority areas without clear documented exposure to TB including hotspots, boarding homes and urban slums. Eligible persons were referred for further screening with chest radiography and follow-on testing with the Xpert MTB/RIF assay. Symptomatic individuals with normal or without radiography results were tested on smear microscopy. We described the TB care cascade and characteristics for each priority group, and calculated yield and number needed to screen. Subsequently, we fitted a mixed-effect logistic regression to identify the association of these target groups and secondary patient covariates with TB treatment initiation.

Results: We verbally screened 321020 people including 24232 household contacts, 3182 social and close contacts and 293606 residents of urban priority areas. This resulted in 1138 persons treated for TB, of whom 85 were household contacts, 39 were close contacts and 1014 belonged to urban priority area residents. The yield of active TB in these groups was 351,1226 and 345 per 100 000, respectively, corresponding to numbers needed to screen of 285, 82 and 290. The fitted model showed that close contacts [adjusted odds ratio $(a O R)=2.07 ; 95 \% \mathrm{Cl}: 1.38-3.11 ; P<0.001$ ] and urban priority area residents $(a O R=2.18 ; 95 \% C l: 1.69-2.79 ; P<0.001)$ had a greater risk of active TB than household contacts.

Conclusions: The study detected a large number of unreached persons with TB, but most of them were not among persons in contact with an index patient. Therefore, while programs should continue to optimize screening in contacts, to close the detection gap in high TB burden settings such as Viet Nam, coverage must be expanded to persons without documented exposure such as residents in hotspots, boarding homes and urban slums.
\end{abstract}

Keywords: Case detection, Tuberculosis, Active case finding, Urban, Viet nam

*Correspondence: luan.vo@tbhelp.org

${ }^{1}$ Friends for International TB Relief, 68B Nguyen Van Troi, 8, Phu Nhuan, Ho Chi Minh City, Viet Nam

Full list of author information is available at the end of the article

\section{Background}

Tuberculosis (TB) remains an intractable public health issue. An estimated 10 million persons develop active TB disease and about 1.6 million people die of TB annually original author(s) and the source, provide a link to the Creative Commons licence, and indicate if changes were made. The images or other third party material in this article are included in the article's Creative Commons licence, unless indicated otherwise in a credit line to the material. If material is not included in the article's Creative Commons licence and your intended use is not permitted by statutory regulation or exceeds the permitted use, you will need to obtain permission directly from the copyright holder. To view a copy of this licence, visit http://creativecommons.org/licenses/by/4.0/. The Creative Commons Public Domain Dedication waiver (http://creativeco mmons.org/publicdomain/zero/1.0/) applies to the data made available in this article, unless otherwise stated in a credit line to the data. 
[1]. Over the past decade, this disease has garnered greater attention and investment [2-6]. This has led to unprecedented levels of TB patients detected and cured. Nevertheless, declines in TB incidence remain too slow to meet global ambitions to end TB by 2030 [7]. A key reason for this slow rate of decline is the "detection gap", defined as the 3-4 million TB patients unreached by national TB control programs (NTP) each year [8]. This gap continues to cause avoidable deaths and fuel transmission of TB [9].

Traditionally, NTPs have relied on passive case finding to detect TB patients. Recognizing that early detection is an essential approach to improve outcomes and abate transmission, the World Health Organization (WHO) recommends systematic screening in key affected populations. However, the number of groups universally recommended for systematic screening remains limited. Strongly recommended groups include household contacts and other close contacts of TB patients, persons living with HIV and persons with silica exposure [10]. Consequently, few persons are actively screened and national programs miss the opportunity to reach more persons suffering from TB [11]. Screening may be expanded to other groups such as urban slum dwellers and migrants, but these recommendations remain conditional [10]. The conditionality derives from the uncertainty that the benefits would outweigh associated costs. This uncertainty is rooted in the lack of documented benefit given the resource intensity of active case finding (ACF) [12]. The paucity of evidence was particularly highlighted among healthcare workers, previously-treated TB patients, and migrants [10].

In Viet Nam, screening for strongly recommended target groups has already been integrated into the country's relevant national guidelines [13-15]. Screening in household contacts is mainly the responsibility of Viet Nam's primary care system. Given that TB care and prevention forms only a minor part of their responsibilities, implementation gaps remain and there is evidence that a significant portion of household contacts that develop active TB are missed by routine NTP activities [16]. However, even if household contact investigations for all publicly notified TB patients were fully implemented, the incremental yield of people with TB would amount to about $1 \%$ of the national TB burden [16].

Similar to many developing countries, there is a growing trend towards urbanization in Viet Nam. This has resulted in an influx of rural-to-urban migrants into major cities [17]. Ho Chi Minh City (HCMC) is a prime destination for those in search of economic opportunity [18]. A consequence is the continuous growth in temporary and urban poor populations [19]. There is substantial evidence that these populations are at greater risk for
TB and poor treatment outcomes [20-22], but it remains unclear whether ACF in these subgroups can yield more case notifications.

Recognizing that routine household contact tracing is both poorly implemented and insufficient to end TB, a community-based ACF project named PROPER CARE was piloted in Go Vap district of HCMC in 2014. This project engaged full-time community health workers (CHWs) for case management and household contact investigation. Project activities further included community-wide door-to-door screening given available $\mathrm{CHW}$ capacity. By 2016, the project had increased case notifications by $17.3 \%$ over baseline. The majority of cases were found among TB patient contacts, or in presumptive hotspots and areas with high concentrations of slum dwellers and migrants [23]. Based on these results we devised the implementing proven community-based active TB case finding interventions study (IMPACT-TB). One of the study goals was to assess screening yield in these urban priority groups in HCMC, Viet Nam.

\section{Methods \\ Study setting}

The study was conducted in six of 24 districts of HCMC (Fig. 1) from October 2017 to September 2019, in which we implemented an ACF intervention. The intervention area was selected through consensus with the provincial TB program and public health authorities based on TB burden, absence of confounding interventions and health system readiness to implement the study. It had a population of 2814034 and notified 4159 all forms TB patients in 2017 [24]. Each district contained one District TB Unit (DTU), which managed TB diagnosis, treatment and notification according to national guidelines. The Pham Ngoc Thach Provincial TB Hospital (PNTH) provided technical supervision, while the Provincial Health Department and District Health Center served as the administrative authorities.

\section{Target populations}

The study targeted three groups for systematic screening: (1) household contacts of index patients; (2) social, community and close contacts of index patients; and (3) residents of urban priority areas. Index patients were persons treated for any form of TB at one of the DTUs of the intervention area and were notified via the national electronic TB recording and reporting system (VITIMES). Household contacts were defined as persons sharing a kitchen with the index case for one or more nights in the past three months prior to treatment initiation of the index case [25]. Social, community and close contacts, collectively referred to as close contacts hereafter, were defined as having interacted with an index patient 


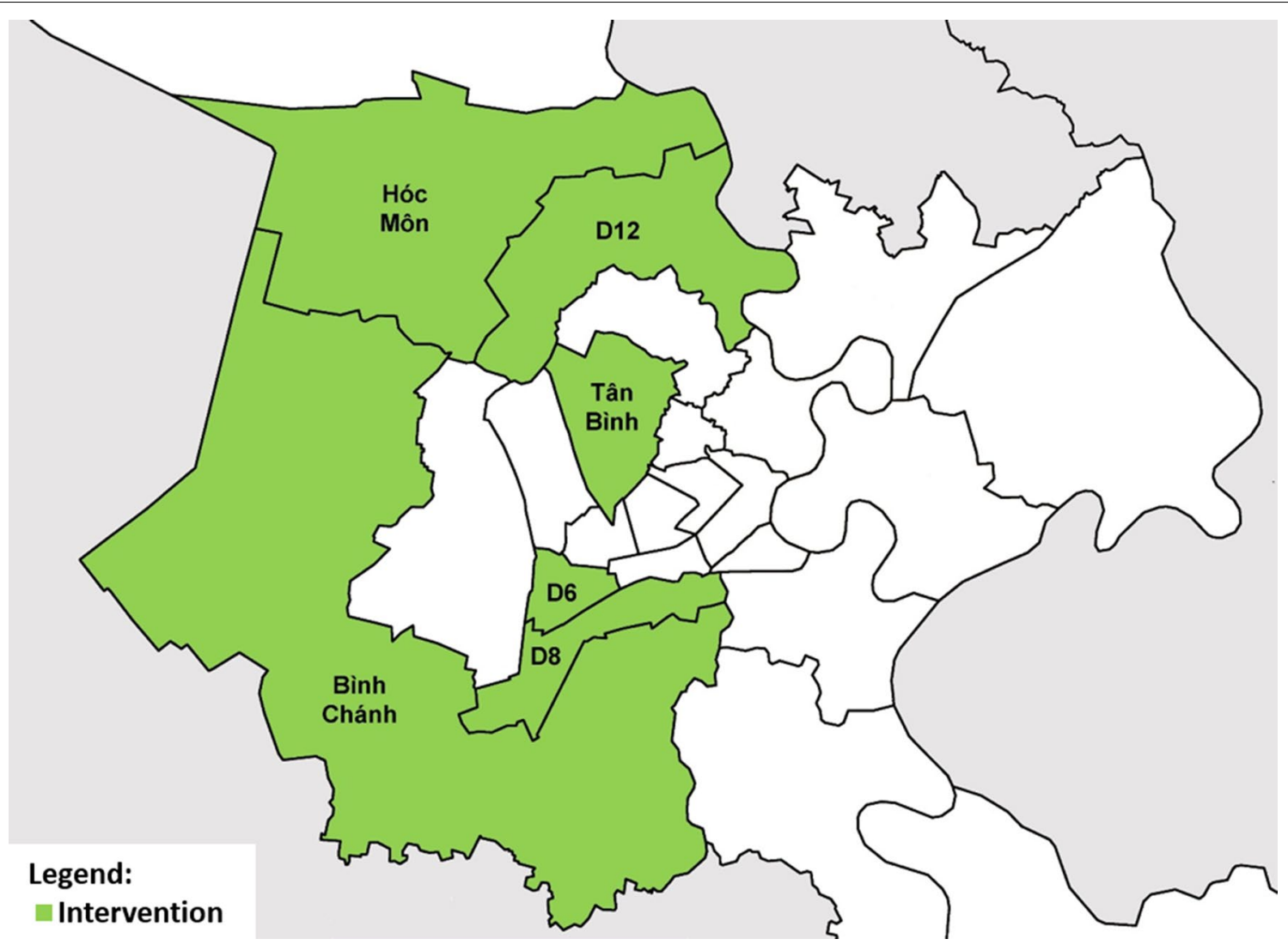

Fig. 1 Location of intervention districts in Ho Chi Minh City, Viet Nam

at least once per month for any duration of time over the past three months and do not meet the definition of household contact [25]. Residents of urban priority areas, referred to as urban priority groups or urban vulnerable populations hereafter, consisted of persons living in proximity of an index case, in a boarding home or in an urban poor community. Proximity was defined as in a $50 \mathrm{~m}$ catchment area of an index case's residence [26] or the same administrative neighborhood (tổ dân phố) as an index case. Boarding homes were defined as dormitories and single-room rental facilities. The definition of urban poor communities was aligned to United Nations Habitat definitions for slum households [27]. District health authorities aided in the identification and prioritization of boarding home and urban poor communities for screening.

\section{ACF intervention}

Each intervention district's local health authorities recruited a cadre of $\mathrm{CHW}$ as incentivized volunteers or salaried employees. The differences of these engagement models have been described elsewhere [28]. These CHWs conducted contact investigation and door-to-door screening. Figure 2 shows the ACF intervention applied in the study by target population. The initial screening step consisted of a verbal screen using a bespoke, Android-based mHealth app (TechUp/Clinton Health Access Initiative, Ha Noi, Viet Nam). Verbal screening included history of TB and symptoms such as (productive) cough, hemoptysis, fever, weight loss, night sweats, dyspnea, chest pain, and fatigue. Consent to participate was requested after the verbal screen and before referral for chest X-ray (CXR) screening. Every household contact was referred for a free CXR at their closest DTU or a weekend screening event regardless of their symptomology; others were only referred for CXR if they reported any one of the aforementioned symptoms, had a history of TB or upon request of the individual. Persons with abnormalities suggestive of TB on CXR were tested on the Xpert MTB/RIF assay (Cepheid; Sunnyvale, CA, USA). Symptomatic persons without CXR results or whose CXR result was normal were tested on smear microscopy. Persons with negative sputum test results were evaluated by the DTU and PNTH for clinical diagnosis in accordance to NTP guidelines. 


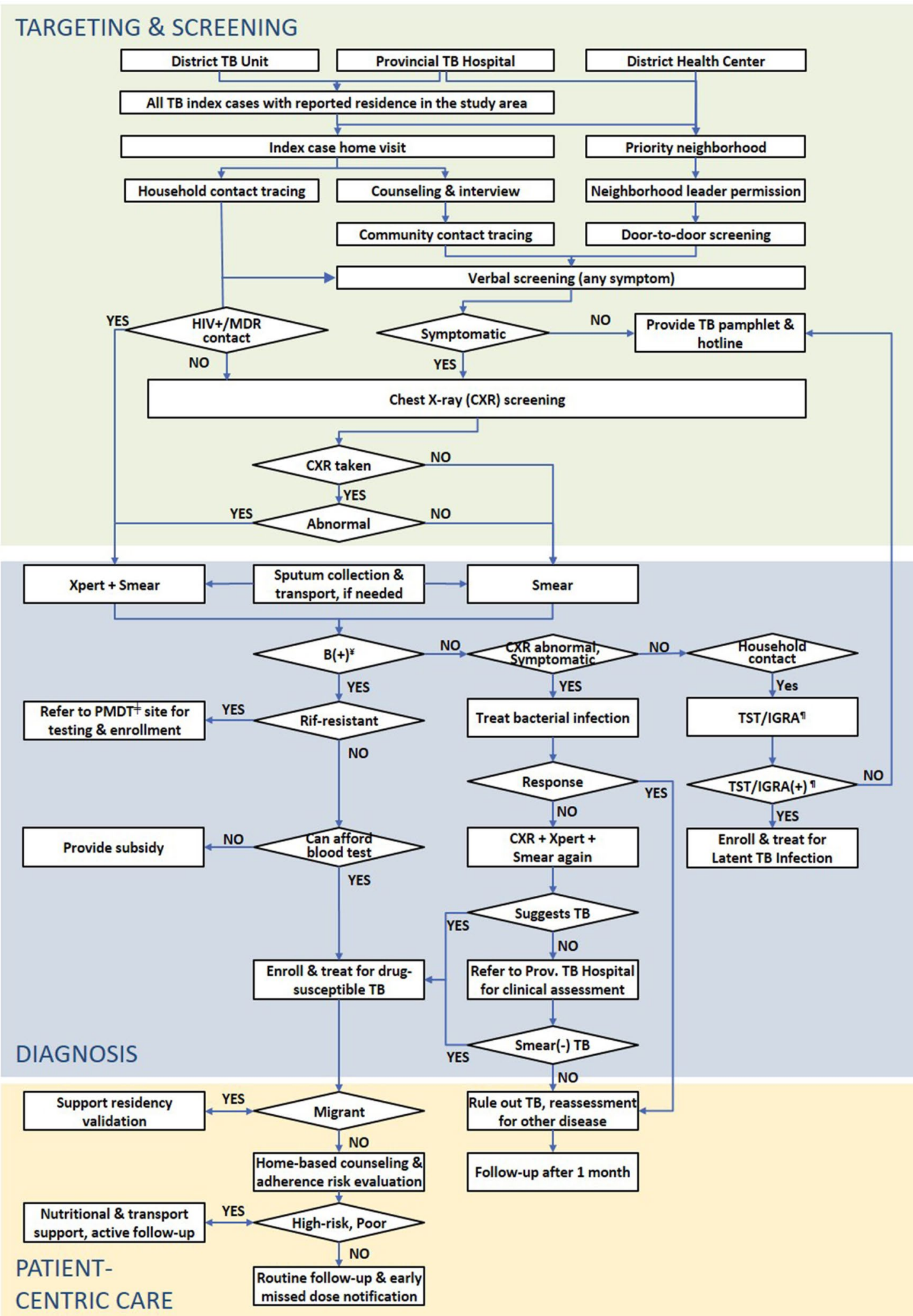

Fig. 2 Active tuberculosis case finding algorithm. TB tuberculosis, MDR multidrug-resistant. ${ }^{¥}$ Bacteriologically-confirmed; ${ }^{\ddagger}$ Programmatic management of drug-resistant TB; "Tuberculin skin test/interferon-gamma release assay 
Persons diagnosed with active TB were initiated on appropriate treatment.

\section{Statistical analyses}

The primary study outcome was TB treatment initiation. Based on this outcome, we calculated the yield and number needed to screen (NNS) for each target group. We constructed a decision tree to illustrate the TB care cascade [29] disaggregated by the three target populations. The secondary study outcome was a risk factor analysis of covariates significantly associated with the primary study outcome among persons referred for CXR. For this analysis, we described number and proportion of persons eligible for CXR screening and fitted a mixedeffect regression to measure the association between TB treatment initiation as the primary outcome and target group as the primary exposure. We included secondary patient covariates to adjust for confounding. The CHW engagement model and district were the random effects to account for intra-cluster correlation. Due to the unexpectedly high NNS among household contacts and low NNS among close contacts, we developed post-hoc analyses for each group. We conducted an aging analysis for household contacts. This analysis described the time elapsed in monthly intervals between TB treatment initiation of the index patient and the subsequent household contact investigation as well as the treatment initiation of another person in the household with TB. We conducted a two-tailed chi-squared test to test for a significant difference between the two groups in each time interval. For close contacts, we disaggregated the number screened, the number treated for active TB disease, and the NNS into friends, relatives, colleagues, neighbors, classmates and services providers. Similarly, we described the age and gender of treated TB patients as a post-hoc analysis due to the lower risk of TB among older participants. Hypothesis tests were two-sided and point estimates included 95\% confidence intervals. Statistical analyses were performed on Stata v13 (StataCorp; College Station, TX, USA).

\section{Ethical considerations}

Ethical approvals were granted by the PNTH Institutional Review Board and the Liverpool School of Tropical Medicine Research Ethics Committee. Study implementation was approved by the HCMC People's Committee. We obtained written informed consent from participants for inclusion in the risk factor analysis and anonymized patient data prior to analysis. Persons that did not consent still received testing and treatment as per the study protocol and NTP guidelines, but were excluded from the analysis.

\section{Results}

Study participants

Our sample included 70439 persons, who consented to be included in the study's risk factor analysis (Table 1). Women comprised $54.5 \%$ of this cohort. The median age was 49 [inter-quartile range (IQR): 33-62]. Most participants lived in urban districts $(63.3 \%)$ and had social health insurance (84.9\%). Approximately $32.1 \%$ had a cough of two weeks or longer, $54.0 \%$ reported at least one of the four main TB symptoms of cough, fever, night sweats and weight loss, and $62.5 \%$ indicated any symptom suggestive of TB. About $5.8 \%$ had a history of TB disease and $7.3 \%$ presented abnormalities on CXR. There were notable differences between the target groups. The proportion of female participants was greater among household contacts (58.0\%) than among close contacts (50.2\%) and urban target groups (52.9\%). The median age among household contacts was lower (37; IQR: 22-53) than among close contacts (44; IQR: 31-57) and urban target groups (55; IQR: 41-65). Urban target groups more frequently reported a cough of at least two weeks (42.7\%) compared to household contacts (13.2\%) and close contacts $(26.0 \%)$. The pattern was similar for other TB symptoms as well. Urban target groups also more commonly had a history of TB (8.1\%) than household contacts (1.9\%) and close contacts $(2.2 \%)$, and showed higher rates of CXR abnormalities (10.2\%) than household contacts (2.2\%) and close contacts (4.1\%).

\section{Active case finding outputs}

The aggregate TB care cascade is in Fig. 3. Overall, 321020 persons were verbally screened and $21.9 \%$ (70 439/321 020) were deemed eligible for CXR referral and were willing to participate, i.e., present for CXR screening. Among those, 62.3\% (43 910/70 439) received a CXR screen and 26.1\% (18 351/70 439) a sputum test. All Forms TB was diagnosed in 1306 participants, of whom $87.1 \%(1138 / 1306)$ were linked to care. This corresponded to a yield of 354 per 100000 in the population screened for a NNS of 282.

The care cascade disaggregated by target population (Fig. 4) shows that over two years the NTP initiated 14365 all forms TB cases on treatment across the six intervention districts. CHWs visited 10989 (76.5\%) households and enumerated 27591 household contacts for an average household size of 3.53 persons. CHWs verbally screened $24232(87.8 \%)$ of the enumerated contacts and 23693 (97.9\%) consented to participate. Active TB disease was diagnosed in $0.4 \%$ (93/24 232) of whom $91.4 \%(85 / 93)$ were linked to care. This yield corresponded to a NNS of 285. During household visits, CHWs further enumerated 3401 close contacts for 
Table 1 Demographic and clinical characteristics of persons screened through active case finding

\begin{tabular}{|c|c|c|c|c|}
\hline & $\begin{array}{l}\text { Total }(n=70439) \\
n(\%)\end{array}$ & $\begin{array}{l}\text { Household contacts } \\
(n=23693) \\
n(\%)\end{array}$ & $\begin{array}{l}\text { Close contacts }(n=2977) \\
n(\%)\end{array}$ & $\begin{array}{l}\text { Urban target } \\
\text { groups }(n=43 \\
769) \\
n(\%)\end{array}$ \\
\hline \multicolumn{5}{|l|}{$\operatorname{Sex}^{\mathrm{a}}$} \\
\hline Male & 31 968/70 211 (45.5) & $9877 / 23498(42.0)$ & $1469 / 2949(49.8)$ & $20622 / 43764(47.1)$ \\
\hline Female & 38 243/70 211 (54.5) & $13621 / 23498(58.0)$ & $1480 / 2949(50.2)$ & 23 142/43 764 (52.9) \\
\hline \multicolumn{5}{|l|}{$\mathrm{Age}^{\mathrm{a}}$} \\
\hline$<15$ years & $4526 / 68986(6.6)$ & $3448 / 22899(15.1)$ & 209/2900 (7.2) & $869 / 43187(2.0)$ \\
\hline $15-29$ years & 9543/68 986 (13.8) & $5061 / 22899(22.1)$ & $462 / 2900(15.9)$ & 4020/43 187 (9.3) \\
\hline $30-44$ years & $14717 / 68986$ (21.3) & $5601 / 22899(24.5)$ & $816 / 2900(28.1)$ & $8300 / 43187$ (19.2) \\
\hline $45-59$ years & $19688 / 68986(28.5)$ & $5509 / 22899(24.1)$ & $831 / 2900(28.7)$ & 13 348/43 187 (30.9) \\
\hline$\geq 60$ years & 20 512/68 986 (29.7) & $3280 / 22899(14.3)$ & $582 / 2900(20.1)$ & 16 650/43 187 (38.6) \\
\hline Median age (IQR) & $49(33-62)$ & $37(22-53)$ & $44(31-57)$ & $55(41-65)$ \\
\hline \multicolumn{5}{|l|}{ Urbanization } \\
\hline Peri-urban & $25854(36.7)$ & 9195 (38.8) & $1383(46.5)$ & $15276(34.9)$ \\
\hline Urban & $44585(63.3)$ & $14498(61.2)$ & $1594(53.5)$ & $28493(65.1)$ \\
\hline \multicolumn{5}{|l|}{ Social health insurance ${ }^{a}$} \\
\hline No & 11 046/70 342 (15.7) & $3806 / 23654(16.1)$ & $641 / 2969(21.6)$ & $6599 / 43719(15.1)$ \\
\hline Yes & 59 296/70 342 (84.3) & $19848 / 23654$ (83.9) & 2328/2969 (78.4) & 37 120/43 719 (84.9) \\
\hline \multicolumn{5}{|l|}{ Cough 2 weeks } \\
\hline No & $47842(67.9)$ & $20559(86.8)$ & $2203(74.0)$ & $25080(57.3)$ \\
\hline Yes & $22597(32.1)$ & $3134(13.2)$ & $774(26.0)$ & $18689(42.7)$ \\
\hline \multicolumn{5}{|c|}{ Four main TB symptoms ${ }^{c}$} \\
\hline No & $32406(46.0)$ & $16682(70.4)$ & $1614(54.2)$ & $14110(32.2)$ \\
\hline Yes & $38033(54.0)$ & 7011 (29.6) & $1363(45.8)$ & $29659(67.8)$ \\
\hline \multicolumn{5}{|l|}{ Any TB symptoms ${ }^{b}$} \\
\hline No & $26406(37.5)$ & $15748(66.5)$ & $1508(50.7)$ & $9150(20.9)$ \\
\hline Yes & $44033(62.5)$ & 7945 (33.5) & $1469(49.3)$ & $34619(79.1)$ \\
\hline \multicolumn{5}{|l|}{ Previous history of TB } \\
\hline No/unknown & $66362(94.2)$ & $23238(98.1)$ & 2911 (97.8) & $40213(91.9)$ \\
\hline Yes & $4077(5.8)$ & $455(1.9)$ & $66(2.2)$ & $3556(8.1)$ \\
\hline \multicolumn{5}{|l|}{ Chest $X$-ray result } \\
\hline No chest X-ray & $26529(37.7)$ & $14274(60.3)$ & $1944(65.3)$ & $10311(23.6)$ \\
\hline Normal & 38804 (55.1) & 8905 (37.6) & $910(30.6)$ & $28989(66.2)$ \\
\hline Abnormal & $5106(7.3)$ & $514(2.2)$ & $123(4.1)$ & $4469(10.2)$ \\
\hline
\end{tabular}

\footnotetext{
a $\mathrm{N}$ sizes listed due to missing values

${ }^{b}$ Includes (productive) cough, hemoptysis, chest pain or dyspnea, fever, night sweats, and fatigue of any duration

c Includes cough, fever, night sweats and weight loss of any duration
}

a rate of 0.3 close contacts per index patient. Of these, $3182(93.6 \%)$ were reached for verbal screening and 2977 (93.6\%) were eligible and consented to participate. Active TB disease was diagnosed in 1.4\% (43/3182) of screened close contacts and 39 (90.7\%) were linked to care for a NNS of 82 . CHWs further reported that 293606 residents of urban priority areas were verbally screened and 43769 (14.9\%) were eligible and consented to be included in the analysis. Active TB disease was diagnosed in 1170 (0.4\%) persons of whom 86.7\%
(1014/1170) were started on treatment for a NNS of 290.

\section{Risk factor analysis}

The adjusted odds ratios are shown in Table 2. Close contacts [adjusted odds ratio $(\mathrm{aOR})=2.07,95 \% \mathrm{CI}$ : $1.38-3.11 ; P<0.001]$ and residents of urban priority areas $(\mathrm{a} O R=2.18,95 \% C I: 1.69-2.79 ; P<0.001)$ were more likely to be treated for active TB compared to household contacts. Coughing for two weeks or longer $(\mathrm{aOR}=2.55$, 


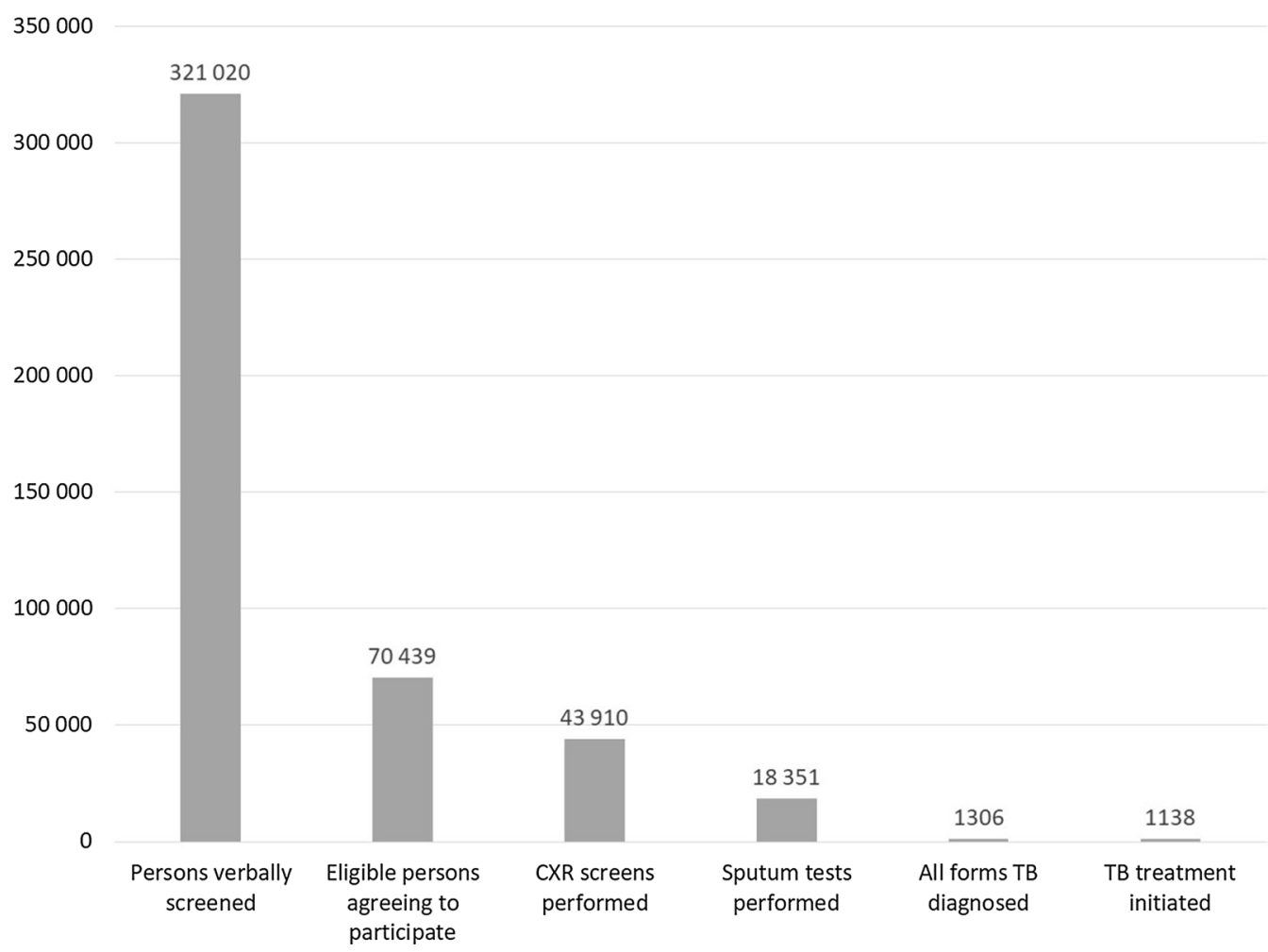

Fig. 3 Aggregate tuberculosis care cascade. CXR chest $X$-ray, TB tuberculosis

95\% CI: 2.12-3.06; $P<0.001)$ and experiencing least one of the four main TB symptoms $(\mathrm{aOR}=1.52,95 \% \mathrm{CI}$ : $1.02-2.26 ; P=0.039$ ) were significantly associated with TB disease. Participants presenting abnormalities on CXR $(\mathrm{aOR}=76.03,95 \% \mathrm{CI}: 58.72-98.44 ; P<0.001)$ and persons without CXR results (aOR $=6.18$, 95\% CI: 4.698.13; $P<0.001$ ) were strongly associated with TB disease in comparison to persons with a normal CXR. Conversely, female participants were significantly less likely to have $\mathrm{TB}$ than male participants $(\mathrm{aOR}=0.55,95 \% \mathrm{CI}$ : $0.48-0.64 ; P<0.001$ ). Using the age group of $30-44$ years as referent, persons under 15 years $(\mathrm{aOR}=0.10,95 \% \mathrm{CI}$ : $0.03-0.32 ; P<0.001)$, between $45-59$ years $(\mathrm{aOR}=0.77$, 95\% CI: $0.65-0.92 ; P=0.003)$ and aged 65 and older $(\mathrm{aOR}=0.41,95 \% \mathrm{CI}: 0.34-0.49 ; P<0.001)$ exhibited a significantly lower association with $\mathrm{TB}$ treatment initiation.

\section{Secondary and post-hoc analyses}

The aging analysis assessing the time interval from index patient treatment initiation to contact investigation (Fig. 5) showed that $66.2 \%$ of TB patient households were visited within the first month after treatment enrollment. Another $18.5 \%$ of patients were visited during the second month of treatment for a total of $84.7 \%$ during the intensive phase. We detected only $40.0 \%$ of TB cases among household contacts within the first two months. Meanwhile, in $35.9 \%$ of household contacts detected with TB, the index case had been treated more than six months prior to the contact tracing event.

Of the 2977 close contacts screened, the index patient had specified the relationship for 2899 (97.4\%) (Table 3). Of the subsequently screened close contacts, 1619 (56.6\%) were neighbors, 831 (23.1\%) were relatives, 236 $(8.3 \%)$ were friends. Among the 39 close contacts treated for TB, neighbors comprised the largest subgroup with 35 TB patients (89.7\%) for an NNS of 46. There were three TB treatment initiations $(7.7 \%)$ among relatives for an NNS of 277. One treated TB case (2.8\%) was a friend of an index case for an NNS of 236.

\section{Discussion}

Our study showed that systematic screening in three urban priority groups using community health workers can yield a substantial number of persons with active TB. We further illustrated the necessity to expand beyond contact investigation to achieve higher coverage and find more people with TB in the community. Nine of ten people diagnosed with TB in this study came from screening in persons without clear prior exposure to TB. The obvious downside was the high number of people that needed 


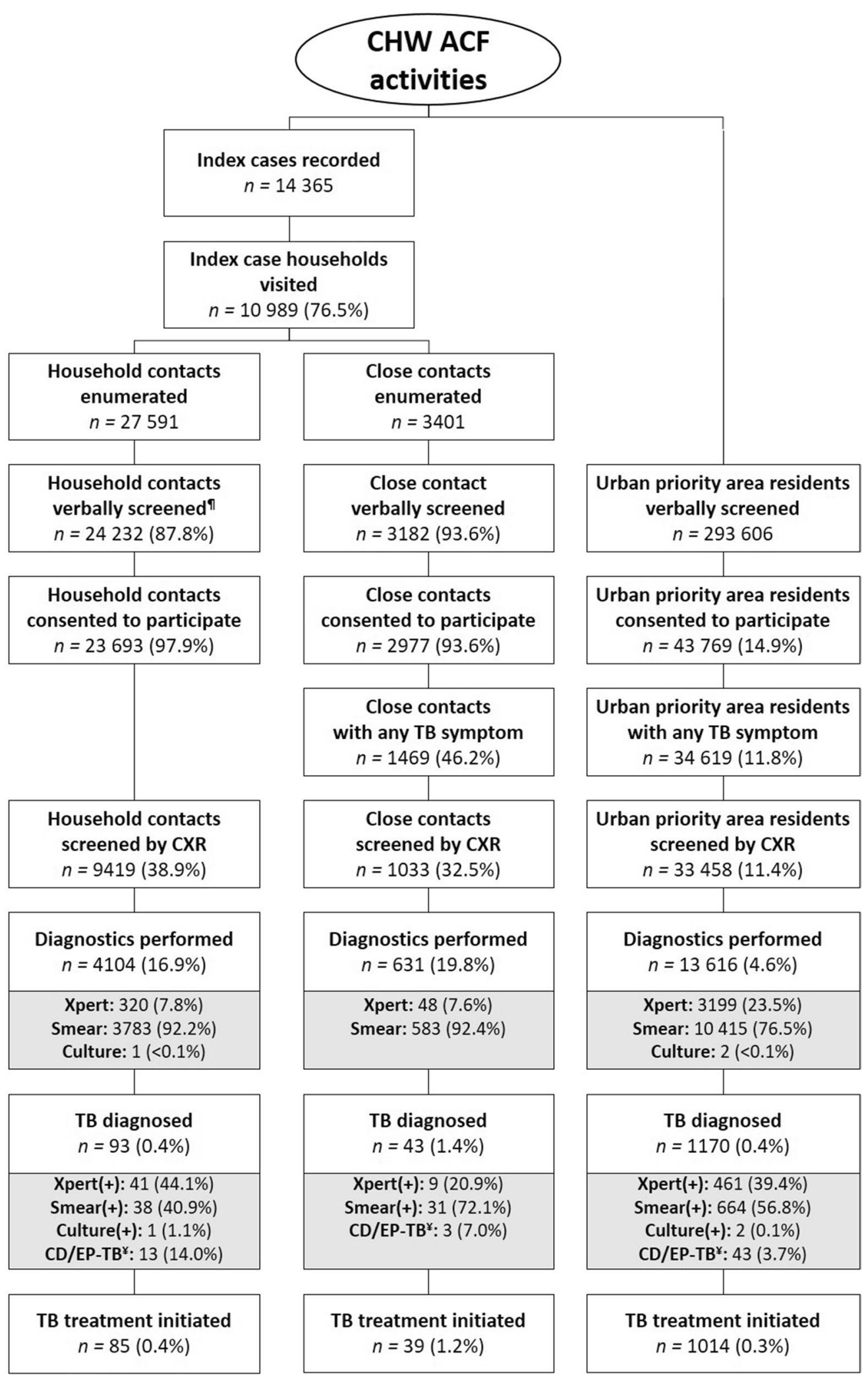

Fig. 4 Tuberculosis care cascade by urban priority group. ACF active case finding, CD clinical diagnosis, CHW community health worker, CXR chest $X$-ray, EP extra-pulmonary, TB tuberculosis. "Verbal screening for household contacts conducted for study-related data collection only; ${ }^{¥} \mathrm{Clinical}$ diagnosed/extrapulmonary tuberculosis 
Table 2 Adjusted associations of urban priority group and secondary covariates with tuberculosis treatment notification from active case finding $(n=68709)$

\begin{tabular}{|c|c|c|c|}
\hline & $\mathrm{aOR}$ & $95 \% \mathrm{Cl}$ & $P$ value $^{\mathrm{p}}$ \\
\hline \multicolumn{4}{|l|}{ Target group } \\
\hline Household contacts ${ }^{*}$ & 1.00 & & \\
\hline Close contacts & 2.07 & $(1.38-3.11)$ & $<0.001$ \\
\hline Urban priority area residents & 2.18 & $(1.69-2.79)$ & $<0.001$ \\
\hline \multicolumn{4}{|l|}{ Sex } \\
\hline Male & 1.00 & & \\
\hline Female & 0.55 & $(0.48-0.64)$ & $<0.001$ \\
\hline \multicolumn{4}{|l|}{ Age } \\
\hline$<15$ years & 0.10 & $(0.03-0.32)$ & $<0.001$ \\
\hline $15-29$ years & 0.94 & $(0.74-1.18)$ & 0.577 \\
\hline $30-44$ years $^{*}$ & 1.00 & & \\
\hline $45-59$ years & 0.77 & $(0.65-0.92)$ & 0.003 \\
\hline$\geq 60$ years & 0.41 & $(0.34-0.49)$ & $<0.001$ \\
\hline \multicolumn{4}{|l|}{ Urbanization } \\
\hline Peri-urban ${ }^{*}$ & 1.00 & & \\
\hline Urban & 1.13 & $(0.93-1.36)$ & 0.211 \\
\hline \multicolumn{4}{|l|}{ Health insurance } \\
\hline $\mathrm{No}^{*}$ & 1.00 & & \\
\hline Yes & 0.87 & $(0.74-1.03)$ & 0.103 \\
\hline \multicolumn{4}{|l|}{ Cough 2 weeks } \\
\hline $\mathrm{No}^{*}$ & 1.00 & & \\
\hline Yes & 2.55 & $(2.12-3.06)$ & $<0.001$ \\
\hline \multicolumn{4}{|l|}{ Four main TB symptoms ${ }^{\S}$} \\
\hline $\mathrm{No}^{*}$ & 1.00 & & \\
\hline Yes & 1.52 & $(1.02-2.26)$ & 0.039 \\
\hline \multicolumn{4}{|l|}{ Any TB symptoms +} \\
\hline $\mathrm{No}^{*}$ & 1.00 & & \\
\hline Yes & 1.46 & $(0.95-2.25)$ & 0.087 \\
\hline \multicolumn{4}{|l|}{ Chest $X$-ray result } \\
\hline Normal* & 1.00 & & \\
\hline Abnormal & 76.03 & (58.72-98.44) & $<0.001$ \\
\hline No chest X-ray & 6.18 & $(4.69-8.13)$ & $<0.001$ \\
\hline \multicolumn{4}{|l|}{ Previous history of TB } \\
\hline $\mathrm{No}^{*}$ & 1.00 & & \\
\hline Yes & 0.90 & $(0.76-1.08)$ & 0.259 \\
\hline
\end{tabular}

aOR adjusted odds ratio, $\mathrm{Cl}$ confidence interval

pWald test

* Referent

+ Includes (productive) cough, hemoptysis, chest pain or dyspnea, fever, night sweats, and fatigue of any duration

$\S$ Includes cough, fever, night sweats and weight loss of any duration

to be screened in order to detect and treat a person with active TB. Even though the NNS in this group (290) was lower than indiscriminate community screening in comparable settings (weighted mean $\mathrm{NNS}=603$ ) [30], this NNS was still on order of magnitude higher than that of strongly recommended groups. Therefore, it is necessary to optimize screening in persons without clear prior exposure to TB or silica, or clinical risk factors such as HIV.

Our study achieved a high yield in close contacts of index cases supporting the recommendation to target this population for ACF. The NNS obtained from our study (82) was similar to previously reported results (weighted mean $\mathrm{NNS}=85$ ) [30]. Our data further showed that index cases most commonly referred neighbors to targeted screening. This group contained nine of ten active TB cases initiated on treatment. This is concordant with prior analysis showing the relevance of neighborhood contacts as potentially vulnerable populations for targeted screening [31, 32]. A prior analysis has shown that the ability to identify hotspots a priori can significantly improve door-to-door screening yield in catchment areas around index cases [26]. As such, door-to-door screening in neighborhoods that house both an index case and a referred neighborhood contact may be a way to sustain high yields while expanding coverage.

However, as only one close contact was enumerated for every three index patients engaged, the coverage achieved among close contacts was limited. A key barrier was the reluctance of index patients to identify others in their social network for targeted screening. Stigma and discrimination were likely reasons for this reluctance [33, 34]. Additional barriers could be the religious and sociocultural context of Buddhism and Confucianism in Vietnamese culture. This cultural context has been associated with passiveness and acceptance of suffering as a normal part of life [35, 36]. Past studies have observed Vietnamese people to hide the extent of their suffering and delay health-seeking [37]. Future close contact tracing should include advocacy tailored to the socio-cultural context to improve participation and referrals [38, 39].

Even though it was more than twice the estimated national incidence rate $(182 / 100000)$ [40], our study produced the lowest yield among household contacts. One reason was the lower rate of TB-related symptoms. This was in part due to the study's diagnostic algorithm, which indicated CXR screening for all enumerated household contacts irrespective of their clinical presentation. As a result, the cohort of household contacts included fewer sick persons and referrals for a CXR were only successful for $39.8 \%$ of household contacts compared to $70.3 \%$ for symptomatic close contacts and $96.6 \%$ of urban target groups. Anecdotal feedback from household contacts substantiated this discrepancy, as these asymptomatic contacts were less motivated to present for CXR screening. Conversely, the high CXR conversion rate in the other two populations was likely a result of the higher value perception of CXR given the positive verbal 


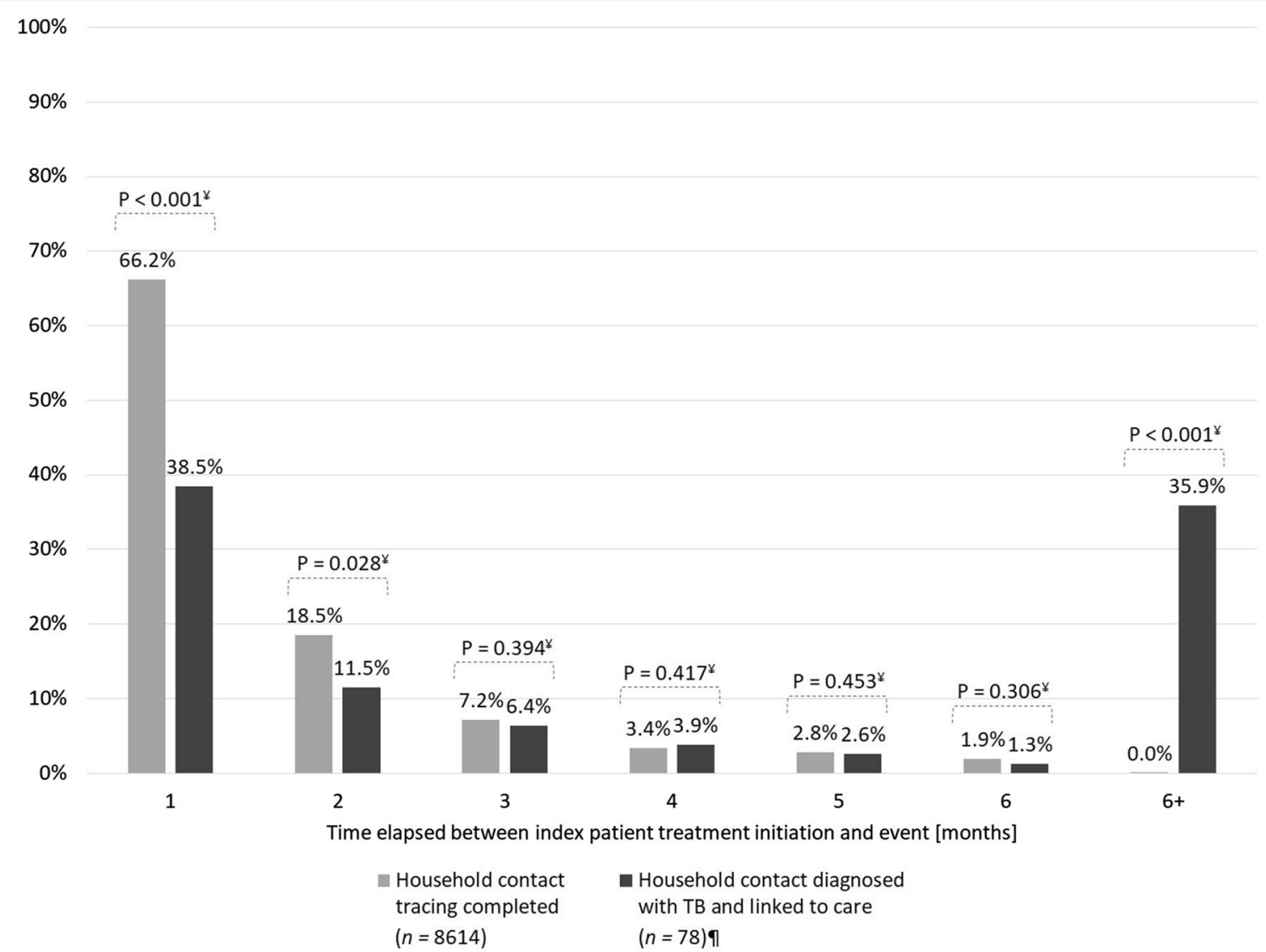

Fig. 5 Time interval from index patient treatment initiation to household contacts investigation and treatment initiation of a household contact with tuberculosis. ${ }^{*}$ Two-tailed $x^{2}$ test; ${ }^{\circledR}$ exact treatment enrollment date for 7 index cases not available

Table 3 Types of close contact screened and treated for active tuberculosis

\begin{tabular}{|c|c|c|c|c|}
\hline & $\begin{array}{l}\text { Screened }{ }^{*}(n=2899) \\
n(\%)\end{array}$ & $\begin{array}{l}\text { Treated for } \mathrm{TB}^{+}(n=39) \\
n(\%)\end{array}$ & $\begin{array}{l}\text { Number needed to screen } \\
n\end{array}$ & $\begin{array}{l}\text { Screening } \\
\text { yield (per } \\
100000 \text { ) }\end{array}$ \\
\hline \multicolumn{5}{|l|}{ Close contact type } \\
\hline Neighbor & $1619(56.6)$ & $35(89.7)$ & 46 & 2162 \\
\hline Relative & $831(29.1)$ & $3(7.7)$ & 277 & 361 \\
\hline Friends & $236(8.3)$ & $1(2.6)$ & 236 & 424 \\
\hline Coworker & $134(4.7)$ & $0(0.0)$ & - & - \\
\hline Service provider & $37(1.3)$ & $0(0.0)$ & - & - \\
\hline Classmates & $3(0.1)$ & $0(0.0)$ & - & - \\
\hline
\end{tabular}

- not applicable

¥ 78 (2.6\%) close contacts missing information on type of relationship

+ All forms tuberculosis (TB), i.e., bacteriologically-confirmed and clinically diagnosed TB patients

pre-screening. While this result shows the value of verbal pre-screening, it may contribute to under-diagnosis of cases. This is particularly the case given the high rate of TB patients without symptomatic presentation as observed on both prevalence surveys [41, 42].
A second reason related to the demographics of screened household contacts. Household contacts were both younger and more often female. Viet Nam's TB prevalence surveys showed that prevalence of TB was lower in younger populations, while older populations are 
another conditionally recommended group for screening [10]. TB prevalence has historically been higher among men [43], which studies have linked to the dichotomy in occupational and behavioral differences across genders in Viet Nam [44], although the complex underlying causes remain insufficiently elucidated.

Another reason for the high NNS in household contacts may relate to the study's contact tracing procedures. CHWs were asked to visit households within one month of treatment initiation to combine contact tracing and adherence counseling. As the aging analysis showed high implementation fidelity, the low yield among household contacts may have been a negative by-product. This effect has been previously observed in Viet Nam. Household contact tracing on treatment initiation yielded only $26.7 \%$ of eventually detected TB cases in the household and at six months the yield was 57.2\% [45]. Our observed yields by time elapsed until contact investigation are concordant with those results. We did not offer TB preventive treatment (TPT) to eligible household contacts. This was a limitation of our approach, due to funding constraints, and future efforts should consider the yield of active TB and simultaneous TPT provision as well as future active TB averted due to TPT provision in their NNS calculations.

Our risk analysis supported the finding that systematic screening should be intensified in close contacts and urban priority area residents. Both groups were more closely associated with the case detected yield in our study. The analysis also showed that cough of two weeks or more remains a strong predictor of TB disease, while the presence of the four main TB-related symptoms was moderately associated with TB disease. Conversely, screening for the presence of additional symptoms such as chest pain and dyspnea was not associated with yield. Similarly, in our study older persons were less associated with case detection yield. This was possibly a function of the door-to-door screening among urban priority populations, which generally occurred during the day. During these screening encounters, CHWs reached a greater proportion of older persons, many of whom did not have TB. Instead, the community-based ACF activities detected a large proportion of working age men with TB (Additional file 1, Table S1), who comprise a key unreached vulnerable population in Viet Nam [42].

CXR abnormality was another strong indicator of risk of TB. This was likely a result of employing the double$\mathrm{X}$ algorithm, which enabled access to the more sensitive Xpert test as the initial diagnostic tool in this cohort compared to smear microscopy in other persons tested. This is concordant with other settings and supports the NTP's country-wide programmatic expansion of the algorithm [46-48]. Even though the relative risk was lower, the cohort without CXR results was also strongly associated with TB treatment initiation compared to persons with a normal CXR. This was likely due to the inclusion of persons who would have presented TB-related abnormalities, had they taken a CXR. As we conducted our study in a programmatic setting, the large number of smear microscopy tests further illustrates the need to broaden access not only to Xpert, but also to CXR in order to justify resource-intensive ACF activities.

The expansion of TB service coverage is critical for closing the detection gap and reaching the End TB goals [49]. Contact investigation undoubtedly should remain the primary adjunct activity to routine program activities. However, the cases detected in this study would have been a fraction without the inclusion of vulnerable groups absent a clear transmission linkage to an index case. Hence, it will be important to conduct studies to optimize and further illustrate the benefits of screening in conditionally recommended priority groups. It will also be necessary to conduct cost-effectiveness assessments that can delineate the trade-offs between the focus on short-term savings and the avoidance of economic loss and human suffering inherent to the long-term elimination of TB.

As the selection of the intervention districts was purposive and limited to urban Ho Chi Minh City, and our study employed convenience sampling of persons screened, our results likely contain bias. Appropriately powered cluster randomized trials would provide a more robust evaluation of these issues. As our study was implemented in a programmatic setting, we were exposed to supply chain interruptions, capacity limitations and restrictive operating hours. These exposures at times impaired adherence to the study's diagnostic algorithm and patient care provision. Furthermore, occasional delays in data entry into the routine surveillance system hindered timely follow-up with index cases. However, these issues reflect the reality of field scale-up of an intervention.

\section{Conclusions}

The study detected a large number of unreached persons with $\mathrm{TB}$, but most of them were not among persons in contact with an index patient, but urban vulnerable populations such as residents of hotspots, boarding homes and urban slums. With a decade remaining until the first major End TB milestone, it is critical to not just optimize screening in strongly recommended target groups such as household and close contacts, but also to expand systematic screening for TB to groups with higher NNS in high TB burden countries such as Viet Nam. Urban priority populations can substantially increase yield and 
urban strategies to end TB. This group should be further considered for inclusion in systematic screening efforts.

\section{Supplementary information}

Supplementary information accompanies this paper at https://doi. org/10.1186/s40249-020-00766-4.

Additional file 1:Table S1. TB treatment notifications by age and sex

\begin{abstract}
Abbreviations
ACF: Active case finding; AFB: Acid-fast Bacilli; aOR: Adjusted odds ratio; CD Clinical diagnosis; CHW: Community health worker; $\mathrm{Cl}$ : Confidence interval; CXR: Chest X-ray; DTU: District TB unit; EP: Extra-pulmonary; HCMC: Ho Chi Minh City; HIV: Human immunodeficiency virus; IQR: Inter-quartile range; NNS: Number needed to screen; NTP: National TB Control Program; PNTH: Pham Ngoc Thach Provincial TB Hospital; TB: Tuberculosis; WHO: World Health Organization.
\end{abstract}

\section{Acknowledgements}

We express our sincere gratitude to the Viet Nam National Tuberculosis Programme, Pham Ngoc Thach Provincial TB Hospital and the DTUs in Districts 1, 2, 3, 4, 6, 8, 12, Tan Binh, Hoc Mon, Binh Chanh, Binh Thanh and Thu Duc for their participation in the study. We thank the field staff of the Ho Chi Minh City Public Health Association. We are deeply grateful for the hundreds of CHWs, who tirelessly worked to support the study, their communities and their patients.

\section{Authors' contributions}

Conceptualization: LNQV, TNV, GTL, GCD, WT, LHN, NVN, BS, KL, MC. Data curation: LNQV, RJF, AJC, NTN, TNV, JL. Formal analysis: LNQV, AJC, JL. Funding acquisition: LNQV, BS, KL, MC. Investigation: LNQV, RJF, AJC, WVT, MC. Methodology: LNQV, RJF, AJC, GCD, HBN, BS, KL, MC. Project administration: LNQV, RJF, AJC, NTN, TNV, GCD, VVT, MC. Supervision: LNQV, GTL, HMD, LHN, HBN, NVN, $B S, K L, M C$. Final approval: all authors have read and approved the manuscript.

\section{Funding}

The IMPACT-TB study and LNQV, AJC, RJF, NTN, TNV, GTL, JL, SBS, KL and MC were supported by the European Commission's Horizon 2020 programme under grant agreement number 733174 . We received additional support from the Stop TB Partnership's TB REACH initiative with funding from the Government of Canada. These funding bodies had no role in the design of the study, in collection, analysis, and interpretation of data, or in writing the manuscript.

\section{Ethics approval and consent to participate}

Approvals were granted by the PNTH Institutional Review Board and the Liverpool School of Tropical Medicine Research Ethics Committee. Study implementation was approved by the HCMC People's Committee. The Pham Ngoc Thach Provincial TB Hospital approved use of the data. We obtained written informed consent from all participants and anonymized all patient data prior to analysis.

\section{Consent for publication}

Not applicable.

\section{Availability of data and material}

The data that support the findings of this study are available from the Viet Nam National TB Control Program and Pham Ngoc Thach Provincial TB Hospital, but restrictions apply to the availability of these data. Data are can be made available from the authors upon reasonable request and with permission of the Viet Nam National TB Control Program and Pham Ngoc Thach Provincial TB Hospital.

\section{Competing interests}

The authors declare that they have no competing interests with regard to this work.

\section{Author details}

${ }^{1}$ Friends for International TB Relief, 68B Nguyen Van Troi, 8, Phu Nhuan, Ho Chi Minh City, Viet Nam. ${ }^{2}$ Interactive Research and Development, Ho Chi Minh City, Viet Nam. ${ }^{3}$ Ho Chi Minh City Public Health Association, Ho Chi Minh City, Viet Nam. ${ }^{4}$ Pham Ngoc Thach Hospital, Ho Chi Minh City, Viet Nam. ${ }^{5}$ National Lung Hospital, Ha Noi, Viet Nam. ${ }^{6}$ KNCV Tuberculosefonds, The Hague, The Netherlands. ${ }^{7}$ Department of Global Public Health, Karolinska Institutet, Stockholm, Sweden. ${ }^{8}$ Department of Clinical Sciences, Liverpool School of Tropical Medicine, Liverpool, UK. ${ }^{9}$ Birat Nepal Medical Trust, Lazimpat, Kathmandu, Nepal.

Received: 14 August 2020 Accepted: 15 October 2020

Published online: 09 December 2020

\section{References}

1. World Health Organization. Global Tuberculosis Report 2018. Geneva: World Health Organization; 2018.

2. World Health Organization. WHO Global Tuberculosis Program communication about the adoption by the UN General Assembly of the Declaration from the first-ever United Nations High Level Meeting on TB. 2019 [cited 2020 Feb 15]. https://www.who.int/tb/features_archive/ UNGA-adopts-TB-declaration/en/.

3. Rusen ID, Enarson DA. FIDELIS—innovative approaches to increasing global case detection of tuberculosis. Am J Public Health. 2006;96:14-6.

4. Stop TB Partnership. Improving tuberculosis case detection: a compendium of TB Reach case studies, lessons learned and a monitoring and evaluation framework. 2014. p. 82.

5. U.S. Government. National Action Plan for Combating Multidrug-resistant Tuberculosis. Washington D.C.: Natl. Strateg. Action Plan Combat. Antibiot. Resist. Bact.; 2015.

6. The global fund to fight AIDS tuberculosis and malaria. Results report 2019. Geneva. 2019.

7. Yuen CM, Amanullah F, Dharmadhikari A, Nardell EA, Seddon JA, Vasilyeva I, et al. Turning off the tap: Stopping tuberculosis transmission through active case-finding and prompt effective treatment. Lancet. 2015;386:2334-43.

8. Stop TB Partnership. The paradigm shift—global plan to end TB: 2018-2022. Geneva; 2018.

9. Kunii O, Yassin MA, Wandwalo E. Investing to end epidemics: the role of the Global Fund to control TB by 2030. Trans R Soc Trop Med Hyg. 2016;110:153-4.

10. World Health Organization. Systematic screening for active tuberculosis: principles and recommendations. Geneva: World Health Organization; 2013.

11. Zhang C, Ruan Y, Cheng J, Zhao F, Xia Y, Zhang H, et al. Comparing yield and relative costs of WHO TB screening algorithms in selected risk groups among people aged 65 years and over in China, 2013. PLoS One. 2017;12(6):e0176581.

12. Biermann O, Lönnroth K, Caws M, Viney K. Factors influencing active tuberculosis case-finding policy development and implementation: a scoping review. BMJ Open. 2019;9:1-12.

13. Viet Nam Ministry of Health. Updated guidelines on the diagnosis, treatment and prevention of tuberculosis, Ha Noi. 2018.

14. Viet Nam Ministry of Health. Circular on treatment management of people infected with HIV and exposed to HIV at health facilities. 28/2018/ TT-BYT, Viet Nam. 2018.

15. Viet Nam Ministry of Health. Guidelines on the diagnosis and recognition of injury due to occupational pneumoconiosis. 36/2014/TT-BYT, Viet Nam. 2014.

16. Fox GJ, Nhung NV, Sy DN, Hoa NLP, Anh LTN, Anh NT, et al. Householdcontact investigation for detection of tuberculosis in Vietnam. N Engl J Med. 2018a;378(3):221-9.

17. Viet Nam General Statistics Office. Migration and Urbanization in Vietnam: Patterns, Trends and Differentials. 2011. https://vietnam.unfpa.org/ webdav/site/vietnam/shared/Censuspublications/7_Monograph-Migra tion-Urbanization.pdf.

18. Le VT. Economic development, urbanization and environmental changes in Ho Chi Minh City, Vietnam: Relations and Policies, Ho Chi Minh City. 2009. 
19. Vietnam General Statistics Office, UNFPA Viet Nam. The 2015 national internal migration survey: major findings. 2016. p 250.

20. Tobe RG, Xu L, Song P, Huang Y. The rural-to-urban migrant population in China: gloomy prospects for tuberculosis control. Biosci Trends. 2011:5:226-30.

21. Duc LV, Vree M, Sy DN, Co NV, Borgdorff MW, Cobelens FGJ. Steep increases in tuberculosis notification among young men in the industrialised districts of Danang, Vietnam. Int J Tuberc Lung Dis. 2007;11:567-70.

22. Vo LNQ, Codlin AJ, Forse RJ, Nguyen HT, Vu TN, Van Truong V, et al. Tuberculosis among economic migrants: a cross-sectional study of the risk of poor treatment outcomes and impact of a treatment adherence intervention among temporary residents in an urban district in $\mathrm{Ho}$ Chi Minh City, Viet Nam. BMC Infect Dis. 2020;20:134.

23. Nguyen TH. Vo Nguyen Quang L, Le TG, Vu NT, Nguyen HD. Results of the community - based intervention for the prevention and control of TB in Go Vap district, Ho Chi Minh city, 2014 [vietnamese]. Viet Nam J Public Health. 2015;38:6-12.

24. Ho Chi Minh City Statistics Office. Population and labour. Ho Chi Minh City: HCMC Statistics Office; 2017.

25. World Health Organization. Recommendations for investigating contacts of persons with infectious tuberculosis in low- and middle-income countries. Geneva: World Health Organization; 2012. p. 28-41.

26. Vo LNQ, Vu TN, Nguyen HT, Truong T, Khuu CM, Pham PQ, et al. Optimizing community screening for tuberculosis: spatial analysis of localized case finding from door-to-door screening for TB in an urban district of $\mathrm{Ho}$ Chi Minh City, Viet Nam. PLoS One. 2018;13:e0209290.

27. UN-Habitat. Slum Almanac 2015/2016: tracking improvement in the lives of slum dwellers. Particip. Slum Upgrad. Program. 2016.

28. Vo LNQ, Forse RJ, Codlin AJ, Vu TN, Le GT, Do GC, et al. A comparative impact evaluation of two human resource models for community-based active tuberculosis case finding in Ho Chi Minh City, Viet Nam. BMC Public Health. 2020;20:934.

29. MacPherson P, Houben RM, Glynn JR, Corbett EL, Kranzer K. Pre-treatment loss to follow-up in tuberculosis patients in low- and lower-middleincome countries and high-burden countries: a systematic review and meta-analysis. Bull World Health Organ. 2014;92:126-38.

30. Shapiro A, Akande T, Lonnroth K, Golub J, Chakravorty R. A systematic review of the number needed to screen to detect a case of active tuberculosis in different risk groups. WHO TB Rev. 2013. https://www.who.int/ tb/Review3NNS_case_active_TB_riskgroups.pdf.

31. Yadav RP, Nishikiori N, Satha P, Eang MT, Lubell Y. Cost-effectiveness of a tuberculosis active case finding program targeting household and neighborhood contacts in Cambodia. Am J Trop Med Hyg. 2014;90:866-72.

32. Gashu Z, Jerene D, Ensermu M, Habte D, Melese M, Hiruy N, et al. The yield of community-based "retrospective" tuberculosis contact investigation in a high burden setting in Ethiopia. PLoS ONE. 2016;11:1-13.

33. Long NH, Johansson E, Diwan VK, Winkvist A. Different tuberculosis in men and women: Beliefs from focus groups in Vietnam. Soc Sci Med. 1999;49:815-22.
34. Tomás BA, Pell C, Cavanillas AB, Solvas JG, Pool R, Roura M. Tuberculosis in migrant populations. A systematic review of the qualitative literature. PLoS One. 2013;8:1-12.

35. Nguyen D. Culture shock - a review of Vietnamese culture and its concepts of health and disease. West J Med. 1985;142:409-12.

36. Jenkins CNH, Le T, McPhee SJ, Stewart S, Ha NT. Health care access and preventive care among Vietnamese immigrants: do traditional beliefs and practices pose barriers? Soc Sci Med. 1996;43:1049-56.

37. Hoang GN, Erickson RV. Cultural barriers to effective medical care among Indochinese patients. Ann Rev Med. 1985;36:229-39.

38. Gibson N, Cave A, Doering D, Ortiz L, Harms P. Socio-cultural factors influencing prevention and treatment of tuberculosis in immigrant and Aboriginal communities in Canada. Soc Sci Med. 2005;61:931-42.

39. Thorson A. Equity and equality case detection of tuberculosis among women and men in Vietnam. Stockholm; 2003.

40. World Health Organization. Global tuberculosis report 2019. Geneva: World Health Organization; 2019.

41. Hoa NB, Sy DN, Nhung NV, Tiemersma EW, Borgdorff MW, Cobelens FG. National survey of tuberculosis prevalence in Viet Nam. Bull World Health Organ. 2010;88:273-80.

42. Nguyen HV, Tiemersma EW, Nguyen HB, Cobelens FGJ, Finlay A, Glaziou $P$, et al. The second national tuberculosis prevalence survey in Vietnam. PLoS One. 2020;15:1-15.

43. United Nations Development Programme. Discussion paper: gender and tuberculosis. 2015.

44. Johansson E, Long NH, Diwan VK, Winkvist A. Gender and tuberculosis control: perspectives on health seeking behaviour among men and women in Vietnam. Health Policy. 2000;52:33-51.

45. Fox GJ, Nhung NV, Sy DN, Hoa NLP, Anh LTN, Anh NT, et al. Householdcontact investigation for detection of tuberculosis in Vietnam. N Engl J Med. 2018b;378:221-9.

46. Philipsen RHHM, Sánchez Cl, Maduskar P, Melendez J, Peters-Bax L, Peter $J G$, et al. Automated chest-radiography as a triage for Xpert testing in resource-constrained settings: a prospective study of diagnostic accuracy and costs. Sci Rep. 2015;5:1-8.

47. Creswell J, Qin ZZ, Gurung R, Lamichhane B, Yadav DK, Prasai MK, et al. The performance and yield of tuberculosis testing algorithms using microscopy, chest x-ray, and Xpert MTB/RIF. J Clin Tuberc Other Mycobact Dis. 2019;14:1-6.

48. World Health Organization. Chest radiography in tuberculosis detection-summary of current WHO recommendations and guidance on programmatic approaches. Geneva: World Health Organization; 2016.

49. World Health Organization. Implementing the end TB strategy: the essentials. Geneva: World Health Organization; 2015. p. 1-130.

Ready to submit your research? Choose BMC and benefit from:

- fast, convenient online submission

- thorough peer review by experienced researchers in your field

- rapid publication on acceptance

- support for research data, including large and complex data types

- gold Open Access which fosters wider collaboration and increased citations

- maximum visibility for your research: over $100 \mathrm{M}$ website views per year

At BMC, research is always in progress.

Learn more biomedcentral.com/submissions 